\title{
Analyse psychologique du mouvement : apport à la compréhension des TMS
}

Psychological analysis of movement: Contribution of musculoskeletal disorders

Análisis psicológico del movimiento y prevención de los tme

\section{Yves Clot et Gabriel Fernandez}

\section{CpenEdition}

\section{Journals}

Édition électronique

URL : http://journals.openedition.org/activites/1818

DOI : 10.4000/activites. 1818

ISSN : 1765-2723

Éditeur

ARPACT - Association Recherches et Pratiques sur les ACTivités

\section{Référence électronique}

Yves Clot et Gabriel Fernandez, « Analyse psychologique du mouvement : apport à la compréhension des TMS », Activités [En ligne], 2-2 I octobre 2005, mis en ligne le 01 octobre 2005, consulté le 30 avri 2019. URL : http://journals.openedition.org/activites/1818 ; DOI : 10.4000/activites.1818

\section{(c) $($ ) $(9)$}

Activités est mis à disposition selon les termes de la licence Creative Commons Attribution - Pas d'Utilisation Commerciale - Pas de Modification 4.0 International. 


\title{
Analyse psychologique du mouvement : apport à la compréhension des TMS
}

\author{
Yves Clot \\ Gabriel Fernandez \\ Équipe clinique de l'activité, CNAM, 41, rue Gay-Lussac, 75005 Paris \\ clot@cnam.fr \\ fernandez@cnam.fr
}

\begin{abstract}
Psychological analysis of movement: Contribution of musculoskeletal disorders

The musculoskeletal disorders (MSD) pose a problem of knowledge and a problem of efficiency of their prevention. From our experiment, we question biomechanics of movement from the psychological analysis of real activity.

The concept of gesture is redefined in order to support the assumption that hypersolicitation of organism results from hyposolicitation of activity. Based on the work of Bernstein, we propose a psychological analysis model of movement postulating that movement, gesture and automatism are three aspects of the same psychophysiological reality. This model allows us to explore the development of the realizable gesture.

We find that the variety of the situations and the development of the repetitions beyond of them self allow the workers to find adequate answers to the motor problems posed by their activity. On the opposite, the impeded development locks up the gesture in identical repetitions, engaging the subjects towards pathogenic compulsive activities. The underdevelopment of the repetitions plunges its roots in the professional activity underdevelopment. The prevention of the MSD should depend on the history of the repetitions by multiplying the gestural experiments and confrontations with the others possible gestures, one's own gestures and those from the others in the collective of work.
\end{abstract}

KEYWORDS

Clinic of activity, Gesture development, prevention of musculoskeletal disorders.

Les troubles musculosquelettiques (TMS) du membre supérieur sont en progression constante depuis plusieurs années. Ils sont souvent à l'origine des demandes d'analyse de l'activité qui nous sont adressées. Les stratégies diagnostiques privilégiant la clinique et une démarche étiologique basée sur la recherche des facteurs professionnels sont désormais courantes pour organiser la prévention de ces affections périarticulaires. Cependant, il faut bien reconnaître que les mécanismes complexes conduisant aux TMS sont encore relativement mal appréhendés du fait des multiples interactions entre les divers facteurs intervenant dans leur genèse.

L'article qui suit se veut une contribution à la discussion ouverte dans cette revue dans son dernier numéro. Nous nous efforçons donc dans cet article d'interroger de manière privilégiée la biomécanique du mouvement à partir de l'analyse psychologique de l'activité réelle. Quelques remarques sur les méthodes d'action viendront à la fin mais ce qui est central ici est la tentative de redéfinir la notion même de geste afin de soutenir l'hypothèse que l'hypersollicitation de l'organisme résulte de l'hyposollicitation de l'activité. 


\section{1.- Geste contrarié et pouvoir d'agir}

Dans la perspective ergonomique qui est la leur F. Bourgeois et F. Hubault (2005) d'un côté et F. Coutarel, F. Daniellou et B. Dugué (2005) de l'autre précisent avec clarté leur position dans la manière d'analyser les TMS. Ils marquent le déplacement nécessaire du regard de la biomécanique du geste vers l'organisation du travail, vers les marges de manœuvres à ouvrir dans la conception et la gestion. Il faut "renseigner" le point de vue économique écrivent Bourgeois et Hubault (2005). La thèse de Coutarel (2004) cherche aussi à instruire simultanément le point de vue de la santé et celui de l'efficacité. Du point de vue de l'économie, la nécessité de ce déplacement des préoccupations vers les contradictions de la gestion et de la conception est confirmée par C. Du Tertre (2005). Dans la nouvelle approche du travail qu'ils proposent aux concepteurs et aux gestionnaires, ces auteurs avancent que l'indicateur de la performance ne peut plus être seulement le geste à faire mais la possibilité du geste à élaborer (Bourgeois, \& Hubault 2005).

Nous partageons cette perspective. Depuis 1995, en retrouvant l'ancienne inspiration de Wallon datant des années 30, nous avons avancé l'idée que c'est l'activité contrariée et pas seulement la contrainte venue du dehors qui est la source des atteintes à la santé en milieu professionnel (Clot, $1995 ; 1997 ; 1998$; 1999). Depuis 1997 , la perspective de clinique de l'activité que nous retenons fait même de l'amputation du pouvoir d'agir une clé pour comprendre les mécanismes délétères agissant dans l'activité des travailleurs (Clot, 1997 ; 1999 ; Clot, Prot, \& Werthe, 2001). Enfin, récemment, nous avons proposé de rapporter l'hypersollicitation des structures musculo-tendineuses, à laquelle on rattache à juste titre les TMS, à une hyposollicitation de l'activité (Clot, 2005 b). Ce programme de recherche en psychologie qui tente depuis 15 ans (Clot, 1990) de tirer le meilleur parti des théories de l'activité (Léontiev, 1984 ;Vygotski, 2003 ; 2005) est au principe de ce qui suit.

Le risque serait en effet, au bénéfice d'une redécouverte des contraintes économiques, de sous-estimer le chemin qui reste à parcourir en ergonomie et en psychologie du travail pour proposer des analyses convaincantes des mécanismes de dé-formation du geste qui font le lit des TMS. La discussion sur les modes d'action et d'intervention sur le geste lui-même reste aussi nécessaire tant elle pose avec acuité le problème de la part que peuvent prendre les travailleurs eux-mêmes à cette action. Nos interventions nous convainquent que le développement de leur pouvoir d'agir sur la situation et sur eux-mêmes passe par l'appropriation subjective qu'ils peuvent faire des méthodes que nous utilisons, c'est-à-dire par le développement de leur rayon d'action dans les interventions que nous conduisons et même sur elles (Clot, 2004 ; Brunet, Presselin, Viel, \& See, 2005).

C'est pourquoi, si on peut dire qu'une clinique de l'activité est toute entière tournée vers l'action, le critère central de l'efficacité de l'intervention est la reconquête de l'initiative sur son propre travail par le collectif d' « opérateurs » concernés, non sans effort de sa part. On peut seconder cette initiative mais sûrement pas la remplacer.

\section{2.- Le développement du geste comme objet de recherche}

Cette orientation délibérée vers la restauration du pouvoir d'agir des collectifs est, simultanément, une expérimentation dont nous cherchons à garantir la portée scientifique en proposant des modèles généraux de l'activité susceptibles d'expliquer ce que d'autres modèles généraux n'expliquent pas. Ce point est pour nous primordial. Il nous conduit par conséquent à risquer une discussion avec la tradition biomécanique. Non pas de l'extérieur, à partir du territoire de l'intervention, ou encore au nom de «facteurs psycho-sociaux », mais de l'intérieur en proposant une alternative à la conceptualisation du geste qui prévaut dans les travaux actuels de biomécanique.

C'est pourquoi une division du travail scientifique, qui partagerait le monde de la recherche sur les TMS entre les partisans d'une démarche explicative et ceux d'une démarche compréhensive, ne 
conviendrait pas complètement à l'objectif que nous nous fixons. À côté de ce que propose l'article très clair de Coutarel, Daniellou et Dugué (2005), nous pensons qu'il y a place pour une voie différente qui regarde l'intervention comme un bon moyen de développer de nouvelles connaissances scientifiques sur le geste et le mouvement.

En ce qui nous concerne, en cherchant à provoquer le développement du pouvoir d'agir des opérateurs sur leurs propres gestes - individuellement et collectivement — dans la situation ordinaire de travail, nous cherchons à expérimenter les possibilités de sortir de l'hyposollicitation de l'activité. Quand les opérateurs y parviennent, l'occasion nous est alors fournie d'étudier, à l'aide d'un modèle d'analyse psychologique du mouvement, un objet scientifique trop rarement exploré : non pas le fonctionnement invariant du geste réalisé mais la dynamique du développement du geste éventuellement réalisable dans le geste réalisé, au-delà du geste réalisé. Si l'on choisit cet objet de recherche concret (Béguin, \& Clot, 2004), l'intervention « compréhensive » cherchant à transformer les gestes à la demande d'un collectif dans une organisation, loin d'être un obstacle à l'« explication » scientifique se présente au contraire comme sa condition. Mieux, elle pourrait bien être un moyen indispensable pour renouveler la théorie du geste (Clot, Fernandez, \& Scheller, à paraître).

\section{3.- Modélisation et biomécanique des TMS}

Quand ils sont d'origine professionnelle, les TMS résultent de contraintes synergiques pesant sur les tissus qui sont alors le siège de réactions dépassant les capacités fonctionnelles individuelles. Les réactions consécutives à ces contraintes sont bien documentées (Richard, \& Orsal, 1994 ; Bouisset, \& Maton, 1996).

La nécessité de les quantifier pour en prévenir les effets, incite à disposer d'un modèle permettant d'évaluer le risque d'apparition des TMS en fonction des contraintes et des capacités fonctionnelles des sujets. Sperling, Dahlman, Wikstrom, Kadefors et Kilbom (1991), Armstrong, Buckle, Fine, Hagberg, Jonsson, Kilbom, et al. (1993), Tanaka et McGlothlin (1993) ont proposé de tel modèles. Considérées comme un tout, les diverses composantes définissent l'exposition dont on cherche à quantifier le niveau, souvent qualifié de dose.

Cependant, à dose comparable, les effets sur les tissus ne sont pas identiques, ce qui se traduit par une grande variabilité intra- et inter-individus. Certains modèles intègrent des perturbations, qualifiées de psychologiques, en lien avec le sujet ou son environnement social, afin de prendre en compte, plus ou moins imparfaitement, les possibilités individuelles de résistance ou d'adaptation face aux différentes doses.

Ces modèles devraient permettre de répondre à des questions de base en prévention : pour une tâche donnée, quel est le niveau de sollicitation biomécanique ? Existe-t-il des différences de sollicitation entre opérateurs pour une même tâche ? L'expérience permet-elle d'exécuter une tâche en réduisant le niveau de sollicitation ? Cependant, des difficultés, liées autant au manque de connaissances portant sur l'intimité des phénomènes biologiques en cause qu'aux problèmes généraux de toute métrologie, limitent pour le moment la portée opératoire de ces modèles.

C'est pourquoi les études épidémiologiques et physiologiques sur les TMS, tout comme les résultats obtenus dans le champ des neurosciences, devraient permettre d'élargir ces modèles « en replaçant le muscle dans sa situation d'effecteur d'un système complexe et intégré, de nature psycho-cognitivo-sensori-motrice » (Aptel, \& Gaudez, 2005) afin de rendre compte, entre autres, des liens biologiques étroits entre le stress et la survenue des TMS d'une part, ainsi que des rapports intimes entre l'activité et ces déterminants biologiques d'autre part. Nous croyons que l'analyse psychologique du mouvement, en en distinguant les différents plans, peut aider à avancer dans cette direction. 


\section{4.- Analyse psychologique du mouvement}

L'analyse que nous proposons s'appuie sur les travaux physiologiques et biomécaniques de Bernstein (1996). Elle a pour but d'établir que mouvement, geste et automatisme sont trois aspects d'une même réalité psycho-physiologique. Nous cherchons à saisir les rapports internes qui lient ces trois instances afin de comprendre la loi du développement des gestes.

Dans cette perspective, nous concevons l'automatisme comme une structure dynamique de l'activité psychophysiologique qui réalise une coordination entre les divers niveaux de contrôle du mouvement.

Le geste est, pour nous, la succession des contractions musculaires, ainsi que leur contrôle et coordination, guidés par un but conscient, assurant le déplacement des segments corporels concernés par ces contractions. C'est l'aspect moteur de l'action.

Le mouvement est la synthèse d'un geste et des régulations posturales nécessaires à porter ce geste. Ces nécessités sont de nature différente : biomécaniques, physiologiques et psychologiques. C'est le niveau intégrateur le plus élevé pour le contrôle de l'exécution aussi bien de l'automatisme que du geste ou du mouvement.

Reprenons chacun de ces aspects. Bernstein a montré que la dextérité du mouvement résulte du développement des gestes. Elle est la capacité de régler les rapports entre l'exécution d'une action et les variations soudaines du contexte de l'action. Cependant, ce n'est pas au niveau du geste que l'on trouve les mécanismes de l'adaptation au contexte, mais à celui des automatismes. Loin d'être des enchaînements invariables de contractions musculaires dont ils prennent parfois l'apparence (Bernstein, 1996), les automatismes allient contractions musculaires variables, contrôle et coordination du mouvement. Ils résultent d'interactions de l'activité d'aires cérébrales transformant les impulsions sensitives en impulsions correctives, expliquant ainsi leur variabilité et leur capacité d'adaptation. L'automatisme réalise l'intégration des dimensions sensitive et motrice, contrôlée au niveau le plus haut de l'activité psychophysiologique. Ce sont les répétitions des gestes dans des contextes que le sujet fait varier qui lui permettent de sentir le mouvement tout en répétant le processus de résolution des problèmes moteurs. Ce dernier consiste à construire les impulsions motrices correctives à partir de la comparaison entre le réalisé et l'attendu, c'est-à-dire, entre la sensibilité de retour produite par le mouvement et la sensibilité produite par la simulation préparatoire du même mouvement au niveau du cortex. Ensuite, la création des synergies réalise la coordination permettant de régler les conflits entre les divers niveaux de contrôle dans leur lutte pour s'approprier les contractions musculaires.

Le geste est une série finalisée de contractions musculaires assurant les déplacements absolus et relatifs des segments corporels impliqués dans l'action en cours. Selon Bernstein, l'activité simultanée de plusieurs centres nerveux, situés à différents niveaux de la hiérarchie neurofonctionnelle, assure le contrôle et la coordination du geste. Le niveau supérieur réalise la coordination en fonction du but poursuivi, tandis que le contrôle est délégué aux centres de niveau inférieur.

La posture, qui consiste à se tenir prêt pour l'action, soutient le geste qui ainsi devient mouvement. Plus précisément, la posture est « un état de préparation à se mouvoir en fonction d'une simulation interne des séquences de mouvement prévues et des buts généraux de l'action. » (Berthoz, 1997, pp. 246-247). Au plan physiologique, les anticipations posturales préparent le geste de telle sorte que les contractions musculaires posturales se fondent avec celles du geste en une synthèse réalisant le mouvement grâce auquel le sujet se mesure au réel.

Le geste n'est disponible en tant que tel qu'en s'extirpant de cette synthèse, non pas par la voie de la destruction du mouvement mais par celle de son développement. C'est en rendant le mouvement d'origine indépendant du premier contexte que le geste devient disponible pour un travail d' «entraînement ». Aucune situation n'existant sans contexte, l' « entraînement » consiste à cons- 
truire une multitude de contextes différents pour y reproduire le geste pris dans un mouvement à chaque fois distinct. Ce processus de décontextualisation et de recontextualisation consistant à accomplir des « répétitions sans répétition » (Bernstein, 1996) est celui du développement du geste.

On peut donc avancer que des gestes différents sont susceptibles de réaliser un même mouvement. Ceci est encore vrai quand deux sujets associent leurs gestes différents et complémentaires dans un mouvement commun (Rouffet, Fraigneau, \& Flores, 2005). A l'inverse, un même geste, par exemple l'extension et l'adduction simultanées du poignet, peut réaliser des mouvements différents comme tirer sur un manipulateur pour serrer les freins d'un train, ou le pousser pour les desserrer (Fernandez, 2004). Nous en concluons que l'activité motrice est un processus unique au sein duquel geste et mouvement ne coïncident pas.

Le geste ne coïncide pas non plus avec les automatismes. Ceux-ci ne sont que les moyens de la réalisation des gestes. Il existe une dimension opérationnelle de l'automatisme expliquant que ce dernier puisse entrer dans la composition de plusieurs gestes différents. À l'inverse, un même geste peut être servi par des automatismes différents, ce qui explique la plasticité fonctionnelle du système moteur.

Ces distinctions nous conduisent à constater qu'entre le geste et le mouvement d'une part, entre le geste et les automatismes de l'autre, il existe la même classe de rapports qu'entre l'action et respectivement l'activité et les opérations dans la théorie de l'activité de Leontiev (1984). Les automatismes réalisent la motricité opérationnelle du geste car celui-ci, conscient et dirigé vers son objet (qui peut être une personne), n'est possible qu'en mobilisant une gamme plus ou moins variée d'automatismes infra-conscients. Le mouvement, quant à lui, engage la posture d'ensemble et l'attitude du sujet. Il est sans doute la composante la plus subjective de l'ensemble. Adressé, il est aussi le résultat de ce qu'un milieu professionnel tient pour adapté ou déplacé. De ce point de vue, il est aussi foncièrement générique et social. C'est à son niveau que sont agissants les régulateurs de l'activité que sont le sens et l'efficience (Clot, 1999), ne serait-ce qu'en raison du fait que toute planification d'un acte moteur est une exploration du futur en cela que le sujet organise des actes non encore réalisés (Berthoz, 1997).

C'est pourquoi nous avons fait l'hypothèse que le corps est le point de rencontre et de transformation du subjectif dans l'organique (Fernandez, 2001). Cependant, cette hypothèse n'est soutenable qu'en considérant que l'organique et le social n'ont pas que des rapports d'extériorité mais qu'ils sont toujours pris dans des processus complexes de transformation de l'un dans l'autre.

Nous avons étayé cette hypothèse grâce aux travaux de Mauss qui a bien vu que l'utilisation du corps est une technique : «le premier et le plus naturel objet technique, et en même temps moyen technique, de l'homme, c'est son corps. » (Mauss, 1934/1997, p. 372). Il faut ici entendre qu'avant d'être un moyen de son activité, l'organisme d'un sujet est un « objet » singulier qu'il doit s'approprier et reconstruire afin de développer de nouvelles fonctionnalités subjectives pour les propriétés organiques. Il faut ajouter que Mauss montre de façon convaincante que cette création, consistant pour un individu à sculpter son organisme pour en faire son corps propre, est simultanément un acte social. S'agissant de l'activité professionnelle, ce modelage de l'organisme est en lien avec les façons de faire en vigueur dans le milieu professionnel, façons que nous rangeons parmi les constituants du genre professionnel (Clot, 1999). Nous voyons aussi l'acquisition de nouvelles gammes gestuelles accompagnant un tel modelage comme le développement de systèmes fonctionnels, au sens que Luria (1993) a donné à ce concept physiologique sur la base des travaux de Bernstein.

Il est d'ailleurs possible de faire un pas supplémentaire dans cette direction. Jeannerod (2005) distingue les mouvements mécaniques des mouvements biologiques produits par des humains par le fait que le mécanisme de ces derniers est double, ce qui leur confère un rôle social. L'observation du mouvement d'autrui permet d'en détecter non seulement les caractères cinétiques mais surtout d'identifier les actions complexes dont il est un des moyens. Les bases neurales de cette propriété 
sont désormais bien établies depuis les travaux de Rizzolatti, Fadiga, Matelli, Bettinardi, Paulesu, Perani et Fazio (1996). Les aires neuronales de la région prémotrice du singe manifestent une activité similaire que l'animal exécute un mouvement ou qu'il le voit faire par l'expérimentateur. La neuro-imagerie a permis de trouver chez l'homme cette même propriété qu'observer une action c'est déjà l'exécuter par anticipation.

Jeannerod remarque que dans la situation d'imitation, l'observateur devenu acteur doit, pour réussir l'imitation, faire correspondre le contenu de l'action afin que la forme externe du mouvement soit conforme au modèle. Ce que prouve l'expérience consistant à montrer un geste raté à un enfant à qui on a demandé d'imiter le mouvement et qui reproduit un geste abouti. Pour Jeannerod, l'enfant de l'expérience ne fait pas que simuler le geste ; il anticipe l'intention de l'acteur qu'il est chargé d'imiter. Il cherche aussi l'intention du geste. Il s'intéresse donc davantage au but qui l'oriente et même à la préoccupation qui l'anime qu'aux moyens de les réaliser.

Metzolff (1995) avait déjà établi des résultats très proches. Plus généralement, en psychologie, ce processus a été minutieusement analysé par Wallon dans ces travaux sur l'imitation (1970). Selon lui, il y a dans l'imitation, plusieurs temps qu'il ne faut pas confondre. Elle est d'abord une action du sujet sur lui-même. Il se fait ainsi l'instrument de ce qu'il voit réaliser près de lui par d'autres et semble s'effacer devant le modèle qui agit en lui. Mais ce modèle, loin de seulement l'appliquer, il le met ensuite au service de sa propre action dirigée non plus sur lui, mais, cette fois, sur le monde. Et du coup, l'imitation incorporee à cette action change de statut. D'abord objet de l'action du sujet sur lui-même, voilà le modèle devenu moyen de son action sur le monde. Le geste-modèle imité se détache alors de la personne imitée. Il devient le geste de l'imitateur, lequel, en retour, comme le note Wallon plus généralement, prend ainsi conscience de lui-même à travers autrui : «C'est en se voulant semblable au modèle qu'il s'oppose à la personne et qu'il doit bien finir par se distinguer aussi du modèle » (1970, p. 157). C'est le troisième temps de l'imitation qui se présente finalement comme un développement possible du geste-modèle si la personne imitée l'autorise, comme un mouvement qui va non seulement du dehors au dedans mais alors aussi du dedans au dehors. Il s'agit là d'un développement de l'imitation, d'une imitation au-delà de l'imitation. Autrement dit le geste naît toujours dans un mouvement partagé avec autrui, échangé, adressé mais aussi littéralement reconstruit, « refait».

\section{5.- Retour à la biomécanique}

L'occasion nous a été donnée d'observer ces distinctions chez des opérateurs d'une chaîne de montage de mécanismes entrant dans la fabrication de disjoncteurs électriques. L'analyse du travail des opérateurs, menée dans le cadre d'une intervention de clinique de l'activité (Clot, Fernandez, \& Scheller, 2003), a commencé par l'enregistrement vidéo de séquences de travail préparant les séances d'autoconfrontations simples et croisées (Clot, 2005 a). Le geste qui permet à un opérateur de saisir une pince pour monter un ressort parvient à son résultat dans tous les cas que nous avons enregistrés. Le résultat du geste est reproductible d'une exécution à l'autre. Cependant, les trajectoires de la main et sa conformation au moment de saisir la pince ne sont jamais exactement identiques d'une fois sur l'autre.

En fait les automatismes que le geste mobilise ne sont nullement identiques à eux-mêmes d'une exécution à l'autre. Leur variabilité est la caractéristique permettant aux opérateurs d'atteindre une excellente performance lors des diverses réalisations du même geste. Le développement des automatismes accroît le nombre de façons différentes de faire le même geste. En quelque sorte, ce développement élargit le nombre de situations possibles dans lesquelles le geste sera efficace. La gamme fonctionnelle du geste s'en trouve enrichie. Au plus cette gamme est riche, au plus l'opérateur peut encore agir efficacement dans des situations imprévues. 
Si l'on suit Bernstein (1996), les automatismes résultent d'une fonction qui dissocie et spécialise les contractions musculaires, de telle sorte qu'elles se modèlent sur la diversité des actions. C'est donc la variété des situations et le développement des répétitions au-delà d'elles-mêmes qui garantissent à l'opérateur une certaine latitude. À l'inverse, dans des situations qui peuvent devenir très anormales, le développement empêché enferme le geste dans des répétitions à l'identique, engageant le sujet vers des activités compulsives où l'aspect moteur n'est plus réalisé par des automatismes mais par des syncinésies. Ces dernières sont un système de mouvements qui ne peuvent s'exécuter qu'ensemble et toujours de la même façon. Cette forme de développement empêché emprisonne les gestes de métiers dans des conduites d'un seul bloc, sorte de gestes «passe-partout », jamais complètement adaptés au problème moteur que l'opérateur cherche à résoudre.

Ce qu'on peut donc appeler le cercle vicieux de la répétition à l'identique, par opposition au cercle vertueux de la répétition sans répétition, entraîne le sous-développement du geste par hyposollicitation du mouvement personnel et collectif. Il assèche et appauvrit le fonctionnement des automatismes en les privant de nouvelles fonctionnalités. Alors, le sujet peut devenir incapable de dissoudre les blocs d'action musculaire pré-établis, dissolution qui lui permettrait de n'utiliser que les combinaisons requises par la situation. Privé de cette plasticité il agit d'un seul bloc.

Nous pouvons donc formuler l'hypothèse que c'est la répétition à l'identique - la répétition compulsive - de la sollicitation des mêmes unités motrices par des syncinésies qui crée une surcharge biomécanique localisée : compression réitérée des structures tendineuses, étirement itératif des mêmes fibres tendineuses ou musculaires au-delà de la limite de rupture. En effet, elle mobilise en toutes circonstances les mêmes structures périarticulaires, faisant ainsi le lit des TMS. C'est qu'alors la répétition au-delà de la répétition est empêchée, contrariée, refoulée. Il nous semble que cette hypothèse pourrait recevoir une confirmation biomécanique. L'importance du contexte ainsi que le remarque Claudon (2005) et surtout de l'activité des sujets pour créer des contextes favorables au développement de leurs gestes mérite plus d'attention. Elle incite à plaider en faveur de méthodologies de recherche où les sujets puissent faire de leurs situations de travail de réelles situations d'expérimentations propres à tester cette hypothèse. La mesure biomécanique pourrait, dans ce cadre renouvelé, se trouver enrichie de fonctionnalités nouvelles et prendre un autre sens. La reprise de l'interrogation par le collectif de travail, à partir des résultats obtenus au moyen de l'étude biomécanique des contraintes opératoires, est déjà envisagée comme un temps indispensable de la stratégie préventive (Rouffet et al., 2005). On peut sans doute aller plus loin dans la perspective d'un développement de l'observation chez les travailleurs eux-mêmes comme instrument d'action.

On retrouve ici les questions de l'action et de l'intervention. Au fond, en clinique de l'activité, ce qui est au centre, ce n'est pas la seule observation mais le développement chez les travailleurs de l'observation de leur propre activité : un changement des protagonistes de l'observation. Mais voilà : pour ce faire, l'observation doit être précise et rigoureusement construite. Le détail devient décisif. Et la mesure peut trouver là de quoi se développer. M. Bakhtine a bien vu le problème : « une observation vivante, compétente, impartiale, à partir d'un point de vue quelconque, garde toujours sa valeur et sa signification. La partialité et la limitation d'un point de vue (d'un observateur), voilà quelque chose qui peut toujours être rectifiée, complétée, transformée (inventoriée) à l'aide de cette même observation à partir d'un point de vue différent » (Bakhtine, 1984, p. 334). A l'inverse, «le point de vue neutralisé (sans observation nouvelle, vivante) est stérile» (idem, p. 334).

On sait que la mesure biomécanique peut être stérilisée si elle est neutralisée, arrêtée. C'est trop souvent le cas. Mais si elle devient un instrument de controverse professionnelle dans le collectif afin de remettre au travail le répertoire générique des gestes professionnels, elle peut aider à prendre la mesure de ce qu'il faut changer dans l'activité et dans l'organisation du travail. En remettant le geste en mouvement dans le dialogue professionnel entre les « connaisseurs » que sont les opérateurs, la mesure biomécanique peut contribuer, à sa place, à l'action de transformation. A une condition : 
qu'elle devienne elle-même un objet d'inventaire, d'examen et de confrontations dialogiques. Mieux, elle peut s'en trouver enrichie, d'autres possibilités de mesures découvertes entre opérateurs et experts pouvant alors être éprouvées par le biomécanicien. C'est la conception de la mesure ellemême qui peut être remise en mouvement. Cette construction sociale de la mesure peut même devenir une ressource pour l'expérimentation biomécanique de laboratoire même si, au départ, elle peut apparaitre d'abord comme un obstacle pour une démarche expérimentale. Sans sous-estimer la difficulté de ces articulations à construire, on doit, selon nous, s'attacher à tisser les liens de cette « communauté scientifique élargie » (Oddone, Rey, \& Briante, 1981).

\section{6.- Conclusion}

Au bout du compte, on retiendra que les TMS sont plus précisément une pathologie du mouvement. Justement classés dans le groupe nosologique des maladies par hypersollicitation liée à une répétition, nous les concevons néanmoins comme le résultat d'un développement pathologique du geste. Nous considérons que le geste est répétitif chaque fois que le mouvement est amputé de ses possibilités de développement. Autrement dit, il est répétitif lorsqu'il ne peut être suffisamment répété dans des contextes hétérogènes. L'hypersollicitation pathogène est alors liée au sous-développement de la répétition, lequel est corrélé à la difficulté, voire à l'impossibilité, que rencontrent les sujets à créer des contextes variés pour y exécuter les gestes professionnels.

Au-delà des sérieuses difficultés techniques de validation des modèles biomécaniques, ces derniers n'intègrent les déterminations psychologiques des TMS que de façon très générale, rendant difficile l'étude expérimentale des interactions entre facteurs de nature différente. L'analyse psychologique du mouvement et de son développement permet de distinguer les différents plans du geste, autorisant une hypothèse à tester concernant ces interactions dont la formulation fait appel à la notion d'hypersollicitation par sous-développement de la répétition.

Selon nous, ce sous-développement plonge ses racines dans celui de l'activité professionnelle. Cela éclaire le fait que les modules de formation «gestes et postures » échouent à prévenir les TMS chaque fois qu'ils sont coupés de l'activité des opérateurs, car la situation réelle est la seule source, seulement potentielle bien sûr, du développement des gestes en mouvements et en automatismes. Tout comme la coopération résulte des controverses entre professionnels sur leur travail, le mouvement résulte de la confrontation des gestes avec les autres gestes possibles, les siens propres ou ceux d'autrui, permettant le développement des automatismes.

Il faudrait donc, pour prévenir les TMS, s'appuyer sur l'histoire des répétitions en multipliant les expériences gestuelles et les confrontations aux autres gestes possibles, ce qui enrichirait la gamme fonctionnelle des automatismes. C'est ce que cherche à réaliser l'intervention en clinique de l'activité (Clot et al., à paraître). Elle ouvre au développement d'autres automatismes en organisant une répétition du mouvement au-delà de lui-même. Elle peut ainsi enrichir la gamme des automatismes, tout comme elle peut le faire des attitudes en explorant le clavier des autres postures possibles. Pour lutter contre l'hyper-sollicitation de l'organisme, elle se porte à la rencontre de l'hypo-sollicitation chronique de l'activité humaine et du corps propre qui anémie les milieux professionnels. Nous avons pris l'habitude de dire et d'écrire en écho aux travaux de nos collègues ergonomes (Coutarel et al., 2005) qu'il faut préserver les marges de manœuvres des «opérateurs ». Pour ce faire, il faut sans doute aussi que ces derniers éprouvent leur capacité propre à manœuvrer les marges. C'est là un objectif central pour une psychologie fondamentale de terrain soucieuse de tirer le meilleur parti des acquis de la pratique ergonomique (Bourgeois, Lemarchand, Hubault, Brun, Polin, \& Faucheux, 2000). Mais paradoxalement on se rapprocherait alors de ce qu'en son temps Bernstein qualifiait joliment de «physiologie de l'initiative » (1996). 


\section{RÉFÉRENCES}

Aptel, M., \& Gaudez, C. (2005). Plausibilité biologique du lien entre les TMS et le stress. Actes du ler Congrès francophone sur les TMS du membre supérieur. Nancy.

Armstrong, T.J., Buckle, P., Fine, L.J., Hagberg, M., Jonsson, B., Kilbom, A., Kuorinka, I.A.A., Silverstein, B.A., Sjogaard, G., \& Viikari-Juntura, E.R. (1993). A conceptual model for work-related neck and upperlimb musculoskeletam disorders. Scandinavian Journal of Work, Environment and Health, 19, 73-84.

Bakhtine, M. (1984). Esthétique de la création verbale. Paris: Gallimard.

Beguin, P., \& Clot, Y. (2004). L'action située dans le développement de l'activité. @ @ctivités, 1 (2), 35-49. http://www.activites.org/v1n2/html/beguin.fr.pdf

Bernstein, N. A. (1996). On dexterity ans its development. In M.L. Latash, \& M.T. Turvey, (Eds). Dextirity and Its Development (pp. 1-235). Mahwah New Jersey: Lawrence. Erlbaum. Associates, Publischers.

Berthoz, A. (1997). Le sens du mouvement. Paris: Odile Jacob.

Bouisset, S., \& Maton, B. (1996). Muscle, posture et mouvement. Paris: Hermann.

Bourgeois, F., \& Hubault, F. (2005). Prévenir les TMS. De la biomécanique à la revalorisation du travail, l'analyse du geste dans toutes ses dimensions. @ ctivités, 2 (1), 37-49. http://www.activites.org/v2n1/ bourgeois.pdf

Bourgeois, F., Lemarchand, C., Hubault, F., Brun, C., Polin, A., \& Faucheux JM. (2000). TMS et travail, quand la santé interroge l'organisation. Lyon: Editions de l'ANACT.

Brunet, R., Presselin, J, Viel, M., \& Sée, N. (2005). Le risque et la parole. Construire ensemble une prévention des risques du travail. Toulouse: Octarès Éditions.

Claudon L. (2005). Problématique de la conception ergonomique d'outils à main. Actes du ler Congrès francophone sur les TMS du membre supérieur. Nancy.

Clot, Y. (1990). Le travail dans l'automobile : du cognitif au subjectif. L'orientation scolaire et professionnelle, 19/4, 311-319.

Clot, Y., (1995). Le travail sans l'homme? Pour une psychologie des milieux de travail et de vie. Deuxième édition 1998. Paris: La Découverte.

Clot, Y. (1997). Le travail, activité dirigée. Contribution à une psychologie de l'action. Thèse d'Habilitation à Diriger des Recherches, Paris: Université de Paris VIII.

Clot, Y. (1998). Le sujet au travail. In J. Boutet, H. Jacot, J. Kergoat, \& D. Linhart (Eds.), Le monde du travail (pp. 287-301). Paris: La Découverte.

Clot, Y. (1999). La fonction psychologique du travail. 4ème édition, 2004. Paris: PUF.

Clot, Y. (2004). Action et connaissance en clinique de l'activité. @ctivités, 1 (1), 23-33. http:// www.activites.org/v1n1/html/clot.pdf

Clot, Y. (2005 a). L'auto-confrontation croisée en analyse du travail : l'apport de la théorie bakhtinienne du dialogue. In, L. Filliettaz, \& J. P. Bronckart (Eds), L'analyse des actions et des discours en situation de travail (pp. 37-55). Bruxelles: De Boeck.

Clot, Y. (2005 b). Les TMS : Hyper-sollicitation ou Hypo-sollicitation? Actes du 1er Congrès francophone sur les TMS du membre supérieur. Nancy.

Clot, Y., Fernandez, G., \& Scheller, L. (2003). Un collectif pour la santé. La prévention des risques de TMS chez Shcneider Electric (AFI-Moirans). Rapport CNAM Paris, Laboratoire de psychologie du travail et de l'action.

Clot, Y., Fernandez, G., \& Scheller, L. (à paraître). Le geste de métier : la transmission comme reconception. Psychologie de l'interaction.

Clot, Y, Prot, B., \& Werthe, C. (2001). Clinique de l'activité et pouvoir d'agir. Education Permanente, $\mathrm{n}^{\circ} 146$.

Coutarel, F. (2004). La prévention des troubles musculo-squelettiques en conception : quelles marges de manoeuvre pour le déploiement de l'activité ? Thèse de doctorat en ergonomie, Université Victor Segalen Bordeaux 2, Editions du Laboratoire d'Ergonomie des Systèmes Complexes. 
Coutarel, F, Daniellou, F., \& Dugué, B. (2005). La prévention des troubles musculo-squelettiques : quelques enjeux épistémologiques. @ ctivités, 2 (1), 3-19. http://www.activites.org/v2n1/coutarel.pdf

Fernandez, G. (2001). Le corps, le collectif et le développement du métier. Étude clinique d'un geste de métier à la SNCF. Éducation permanente, 146, p. 27-35.

Fernandez, G. (2004). Histoire d'un geste : le cas du freinage d'un train en Gare du Nord. Thèse de Doctorat en Psychologie. Paris: CNAM.

Jeannerod M. (2005). Le cerveau intime. Paris: Odile Jacob.

Leontiev, A. (1984). Activité, conscience, personnalité. Moscou: Editions du Progrès.

Luria, A. R. (1993). Epilogue. In L.S. Vygotski (Ed.), Obras escogidas. Tome 2 (pp. 451-470). Madrid: Visor/ Aprendizaje.

Mauss, M. (1934/1997). Sociologie et anthropologie. Paris: PUF.

Metzolff, A. N. (1995). Understanding the intentions of others : Re-enactment of intended acts by 18 -monthold children, Developmental Psychology, 31, 212-220.

Oddone, I., Rey, A., \& Briante, G. (1981). Redécouvrir l'expérience ouvrière. Vers une autre psychologie du travail. Paris: Editions Sociales.

Richard, D., \& Orsal, D. (1994). Neurophysiologie. Organisation et fonctionnement du système nerveux. Paris: Dunod.

Rizzolatti, G., Fadiga, L., Matelli, M., Bettinardi, V., Paulesu, E., Perani, D., \& Fazio, F. (1996). Localization of grasp representations in humans with PET. 1. Observation versus execution. Experimental Brain Research, 111, 246-252.

Rouffet, D., Fraigneau, F. \& Flores, J-L. (2005). Influence de deux modes d'exécution du travail sur les astreintes biomécaniques. Actes du 1er Congrès francophone sur les TMS du membre supérieur. Nancy.

Sperling L., Dahlman S., Wikstrom L., Kadefors R., \& Kilbom A. (1991). Tools and hand function: the cube model. A method for analysis of the handling of hand tools. In Y. Quéinnec Y., \& F. Daniellou (Eds). Designing for everyone. Proceedings from the 11th Congress of the International Ergonomics Association, Paris, vol. 1 (pp. 176-178). London, Taylor et Francis.

Tanaka S., \& McGlothlin J.D. (1993). A conceptual model for prevention of work-related carpal tunnel syndrome (CTS). International Journal of Industrial Ergonomics, 11, 181-193.

Tertre du, C. (2005). Services immatériels et relationnels : intensité du travail et santé. @ ctivités, 2 (1), 37-49. http://www.activites.org/v2n1/dutertre.pdf

Vygotski, L. (2003). Conscience, inconscient, émotions. Paris: La Dispute.

Vygotski, L. (2005). Psychologie de l'art. Paris: La Dispute.

Wallon, H. (1970). De l'acte à la pensée. Paris: Flammarion.

\section{RÉFÉRENCEMENT}

Clot, Y., \& Fernandez, G. (2005). Analyse psychologique du mouvement : apport à la compréhension des TMS. @ ctivités, 2 (2), 69-78, http://www.activites.org/v2n2/fernandez.pdf

\section{RÉSUMÉ}

Les troubles musculosquelettiques (TMS) posent un problème de connaissance doublé d'un problème d'efficacité de leur prévention. à partir de notre expérience de terrain, nous interrogeons la biomécanique du mouvement du point de vue de l'analyse psychologique de l'activité réelle.

La notion de geste est redéfinie afin de soutenir l'hypothèse que l'hypersollicitation de l'organisme résulte de l'hyposollicitation de l'activité. En nous appuyant sur les travaux de Bernstein, nous proposons un modèle d'analyse psychologique du mouvement postulant que mouvement, geste et automatisme sont trois aspects d'une même réalité psycho-physiologique. Ce modèle nous permet d'explorer le développement du geste réalisable. 
Nous trouvons que la variété des situations et le développement des répétitions au delà d'elles-mêmes permettent aux travailleurs de trouver des réponses adéquates aux problèmes moteurs posés par leur activité. à l'inverse, le développement empêché enferme le geste dans des répétitions à l'identique, engageant les sujets vers des activités compulsives pathogènes. Le sous-développement des répétitions plonge ses racines dans celui de l'activité professionnelle. La prévention des TMS devrait s'appuyer sur l'histoire des répétitions en multipliant les expériences gestuelles et les confrontations aux autres gestes possibles, les siens et ceux d'autrui dans le collectif de travail.

\section{MOTS-CLÉS}

Clinique de l'activité, développement du geste, prévention des Troubles Musculo-Squelettiques.

\section{RESUMEN}

Análisis psicológico del movimiento y prevención de los tme;

Los trastornos músculo-esqueléticos (TME) plantean un problema de conocimiento acrecentado, a su vez, por la eficacia de su prevención.

Partiendo de nuestra experiencia de campo, y adoptando el enfoque del análisis psicológico de la actividad real, interrogamos a la biomecánica del movimiento. Y, para poder sostener la hipótesis según la cual la hiper exigencia del organismo es la resultante de la sub exigencia de la actividad, redefiniremos la noción de gesto. Apoyándonos en los trabajos de Bernstein, proponemos un modelo de análisis psicológico del movimiento que postula que movimiento, gesto y automatismo resultan ser tres aspectos de una misma realidad psico-fisiológica y que nos permite explorar el desarrollo del gesto a realizar. Tanto la variedad de situaciones como el desarrollo de las repeticiones permiten a los trabajadores, más allá de las mismas, encontrar respuestas adecuadas a los problemas motrices planteados por su actividad. Inversamente, el desarrollo impide y encierra al gesto en repeticiones idénticas, llevando a los sujetos hacia actividades compulsivas y patógenas. El subdesarrollo de las repeticiones hunde sus raíces en el de la actividad profesional. Y la prevención de los TME debería apoyarse en la historia de las repeticiones, multiplicando las experiencias gestuales y las confrontaciones con otros gestos posibles, los propios y los de los otros en el colectivo de trabajo.

PALABRAS CLAVE

Clínica de la actividad, desarrollo del gesto, prevención de los Trastornos Músculo-Esqueléticos. 